\title{
|||||||||||||||||||||||||||||||||||||||||||||||||||||||||||||||||||.
}

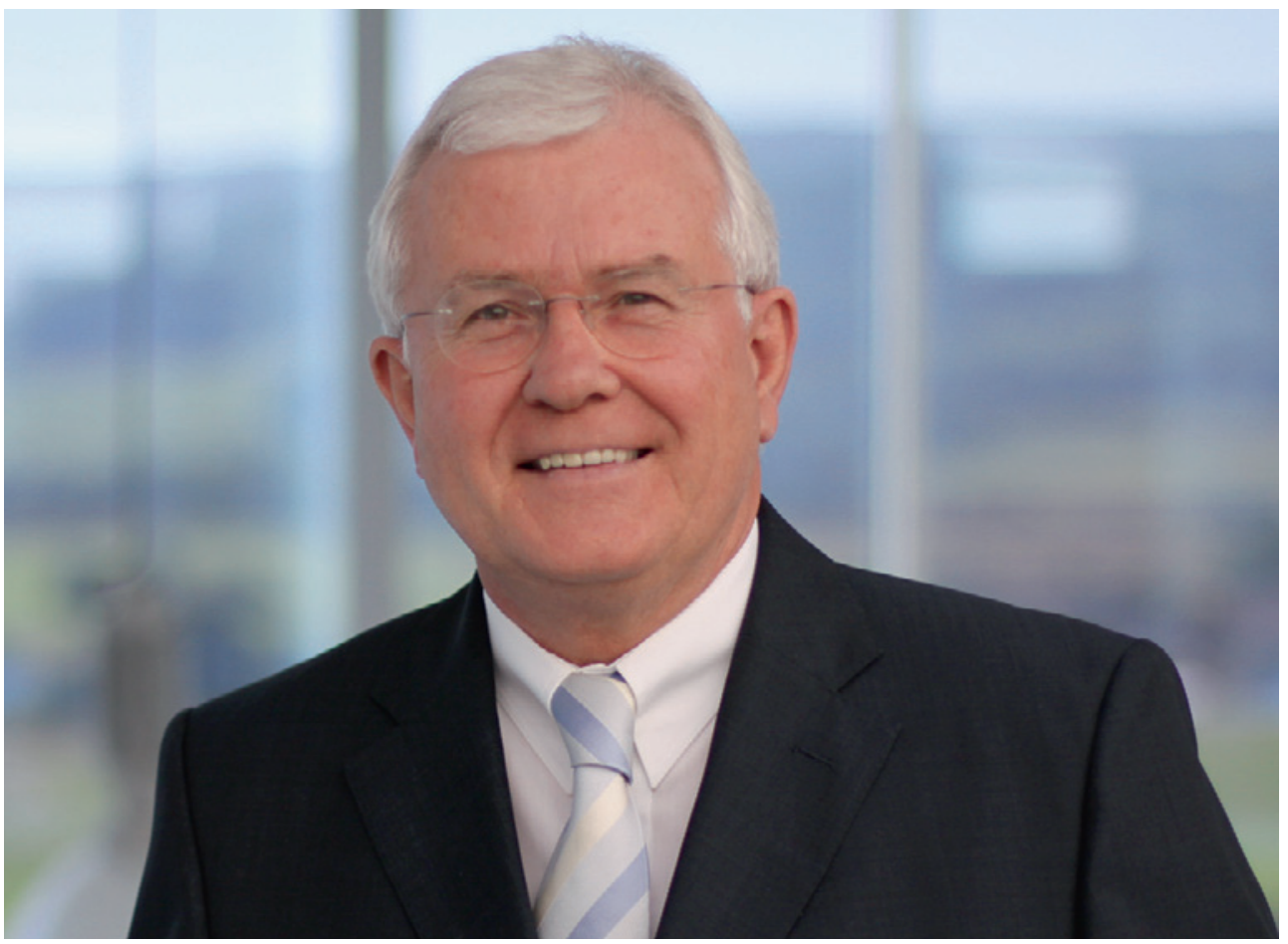

DR. RER. OEC. KURT JONAS

Geschäftsführer

Ricardo Deutschland

\section{AUFGABEN DER KOMMENDEN DEKADEN}

Es sind drei hauptsächliche Herausforderungen, denen sich die Automobilindustrie in den nächsten Dekaden stellen muss. Es gilt, Fahrzeuge anzubieten, die erstens Mobilität mit vernachlässigbarer Emission von Treibhausgasen ermöglichen, zweitens weiter erhöhte aktive und passive Sicherheit bieten und drittens nachhaltig produziert werden können.

Der Antriebsstrang, ob im motorisierten Zweirad, in einem Pkw oder im Nutzfahrzeug, wird eine Schlüsselrolle bei der Meisterung dieser Herausforderungen spielen. Die Elektrifizierung des Triebstrangs war im letzten Jahrzehnt ein Schwerpunkt der Entwicklungsanstrengungen und wird auf absehbare Zeit ein bedeutender Trend bleiben. Eine Betrachtung des gesamten Fahrzeugmarkts legt aber nahe, die Elektrifizierung als ein Kontinuum vom MikroHybrid mit konventionellem Verbrennungsmotor bis zum rein elektrischen Fahrzeug zu sehen. Am „elektrischen Ende“ dieses Kontinuums sind dabei die Aufgaben der kosteneffektiven Energiespeicher und der „durchgereichten Emission“ zu bewältigen.

Die Elektrifizierung wird auch weitere Chancen für die zukünftige Entwicklung der Getriebetechnik eröffnen. Trotz der jüngeren, schnellen Fortschritte bei wachsender Komplexität sollte man die Elektrifizierung als einen Weg sehen, Funktionen zu kombinieren und zu integrieren und dabei auf Vereinfachungen abzuzielen. Das Getriebe mit elektrischen Antriebskomponenten zu kombinieren, die zusätzlich die Nebenaggregate versorgen, während der Motor steht, wird eine wichtige Zukunfts- aufgabe sein. Die Integration von Motor- und Getriebesteuergerät in ein Steuergerät für den vollständigen Triebstrang wird von nicht geringerer Wichtigkeit sein, wobei Kombination und Integration hier als Methoden zur Steigerung des funktionalen Leistungsumfangs bei gleichzeitiger Verringerung des erforderlichen Applikationsaufwands gesehen werden sollten.

Letztlich sollte der Verbrennungsmotor nicht vergessen werden. Sein Wirkungsgrad weist noch erhebliches Steigerungspotenzial auf, und das Ziel für einige Straßenfahrzeuge sollte durchaus auf $60 \%$ effektiv im Bestpunkt gesteckt werden. Bei Pkw sehen wir eine Konvergenz der typischen Eigenschaften von Diesel- und Ottomotoren. Der Kraftstoffverbrauch der Ottomotoren bewegt sich dank Magerbetrieb und Direkteinspritzung auf das Dieselniveau zu, während die Literleistung der Dieselmotoren immer mehr steigt und so weiteres Downsizing und Downspeeding ermöglicht. In Verbindung mit weiterentwickeltem Thermomanagement, einschließlich Wärmerückgewinnung zur Konversion und Speicherung, werden Verbrennungsmotoren mindestens bis 2030 die dominierenden Antriebsmaschinen bleiben.

Der Antriebsstrang wird sich in Zukunft nicht nur durch weitere, signifikante Verbesserungen seiner Komponenten entwickeln, sondern auch durch die konsequente Integration derselben, um so den Herausforderungen der nächsten 30 Jahre gerecht zu werden. 